\title{
Postural lung recruitment assessed by lung ultrasound in mechanically ventilated children
}

\author{
Gerardo Tusman ${ }^{1 *}$, Cecilia M. Acosta ${ }^{1}$, Stephan H. Böhm², Andreas D. Waldmann ${ }^{3}$, Carlos Ferrando ${ }^{4}$, \\ Manuel Perez Marquez ${ }^{5}$ and Fernando Suarez Sipmann ${ }^{6,7,8}$
}

\begin{abstract}
Background: Atelectasis is a common finding in mechanically ventilated children with healthy lungs. This lung collapse cannot be overcome using standard levels of positive end-expiratory pressure (PEEP) and thus for only individualized lung recruitment maneuvers lead to satisfactory therapeutic results. In this short communication, we demonstrate by lung ultrasound images (LUS) the effect of a postural recruitment maneuver (P-RM, i.e., a ventilatory strategy aimed at reaerating atelectasis by changing body position under constant ventilation).

Results: Data was collected in the operating room of the Hospital Privado de Comunidad, Mar del Plata, Argentina. Three anesthetized children undergoing mechanical ventilation at constant settings were sequentially subjected to the following two maneuvers: (1) PEEP trial in the supine position PEEP was increased to $10 \mathrm{cmH}_{2} \mathrm{O}$ for 3 min and then decreased to back to baseline. (2) P-RM patient position was changed from supine to the left and then to the right lateral position for $90 \mathrm{~s}$ each before returning to supine. The total P-RM procedure took approximately 3 min. LUS in the supine position showed similar atelectasis before and after the PEEP trial. Contrarily, atelectasis disappeared in the non-dependent lung when patients were placed in the lateral positions. Both lungs remained atelectasis free even after returning to the supine position.
\end{abstract}

Conclusions: We provide LUS images that illustrate the concept and effects of postural recruitment in children. This maneuver has the advantage of achieving recruitment effects without the need to elevate airways pressures.

Keywords: Children, Airways, Outcome, Respiration, Lung ultrasound, PEEP, Anesthesia-induced atelectasis, Lung recruitment, Mechanical ventilation

\section{Background}

The incidence of atelectasis in mechanically ventilated children with healthy lungs is as high as $68-100 \%$ [1-4]. Anesthesia-induced atelectasis has been well described by different imaging techniques such as CT, MRI and lung ultrasound (LUS) [1-5]. The main mechanism leading to atelectasis formation during anesthesia is a decrease in functional residual capacity due to lung compression through the dysfunctional diaphragm by abdominal content [6,7]. During such conditions, the

\footnotetext{
*Correspondence: gtusman@hotmail.com

1 Department of Anesthesiology, Hospital Privado de Comunidad,

7600 Mar del Plata, Buenos Aires, Argentina

Full list of author information is available at the end of the article
}

trans-pulmonary pressure (Ptp = airways - pleural pressure) is no longer sufficient to offset these compressive forces on the most gravity-dependent parts of the lungs, where Ptp is naturally the lowest. Compression atelectasis has clearly been demonstrated in anesthetized children and adults $[3,7-10]$.

\section{Rationale of postural lung recruitment}

Lung recruitment maneuvers are ventilator strategies that elevate airway pressures for a few breaths to reaerate atelectasis $[11,12]$. Such maneuvers are safe and easily to conduct in mechanically ventilated children $[3,13]$. However, potential hemodynamic side effects of high intrathoracic pressures necessitate close hemodynamic 
monitoring and that such maneuvers are performed in normovolemic patients, only [12].

In this short communication, we explain the rationale of a new type of lung recruitment maneuver-the postural recruitment (P-RM). It can be defined as an active reaeration of atelectatic lung tissue by intentional changes of a patient's body position under constant ventilatory conditions. This concept makes use of the influence that gravity has on respiratory physiology, more precisely on Ptp. The superimposed pressure within the thorax caused by the lung's own weight decreases Ptp by approximately $0.25 \mathrm{cmH}_{2} \mathrm{O}$ for every $1 \mathrm{~cm}$ of ventral-to-dorsal thoracic diameter. Therefore, in supine mechanically ventilated patients Ptp decreases along the gravitational axis causing the lungs to collapse in their most dorsal parts (lowest Ptp) while keeping the ventral zones (highest Ptp) aerated and thus "open" during the entire respiratory cycle [12]. No matter in which body position the patient is placed, dorsal lung zones will always be prone to atelectasis and airways closure $[6,7,10]$.

Considering this gravity-dependent physiology, the additive effect of the two following principles can be used therapeutically to recruit lungs by intentionally changing the body position of ventilated patients:

- The first principle refers to the relationship between Ptp and body position. It postulates that collapsed tissue in dependent lung zones can be recruited by placing the patient in the opposite position (i.e., from supine to prone or from the left to the right lateral decubitus position). This way, previously collapse lung tissue is now located in the non-dependent position where it is being subjected to the expanding forces of higher Ptps.

- The second principle refers to the stabilizing effect of positive end-expiratory pressure (PEEP) on airways and alveoli. It postulates that-provided sufficient PEEP is applied-well ventilated and fully aerated non-dependent lung areas will remain "open" even when becoming dependent again after returning the body to its initial position.

Based on the above principles, we propose a postural recruitment maneuver in which a supine patient is first turned onto his left, then onto his right side and finally to his original supine position (Fig. 1). Thus, we speculate that lung recruitment can be achieved by postural changes without having to elevate airway pressures [11, 12].

Mechanically ventilated children are a sub-population that could especially benefit from this intervention for two reasons: (1) the respiratory physiology of pediatric patients makes them more susceptible to lung collapse after tracheal intubation than adults [8,9], and (2) children are usually of light weight and can thus be easily turned by one single operator.

In this short communication, we present lung ultrasound (LUS) images which confirm the recruitment effect of intentional changes of body position in anesthetized children. LUS is a good non-invasive tool for

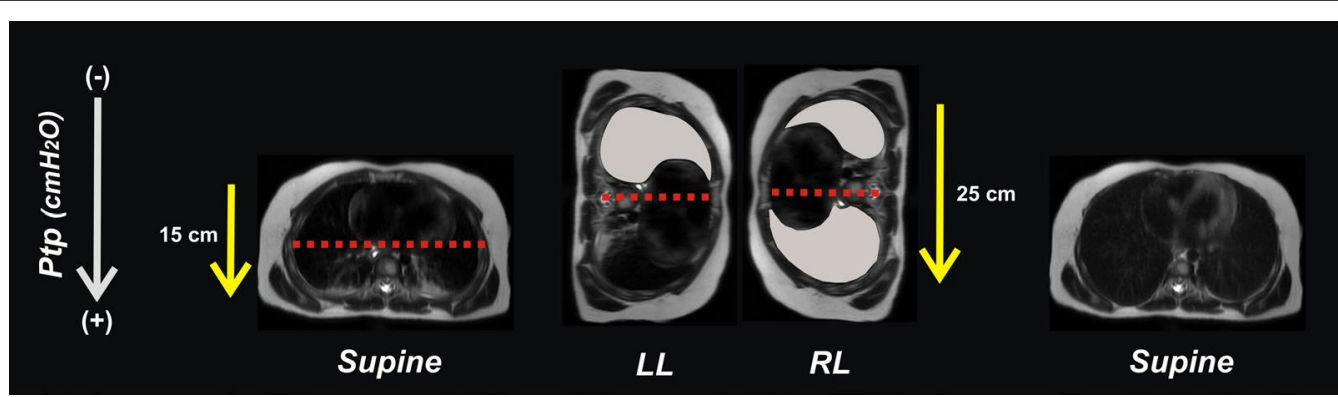

Fig. 1 Concept of postural lung recruitment. Theoretical explanations of the postural recruitment maneuver in an anesthetized child 6 years of age. The maneuver consists of sequential changes in body position from supine to both lateral positions and back to supine again, keeping ventilatory settings constant. The gradient of trans-pulmonary pressures (Ptp) differs between body positions due to the elliptical shape of the chest with the gravity-dependent thoracic dimension being larger in lateral than in the supine position (yellow arrows). Thus, the lower half of the lungs is predisposed to collapse while the upper half is usually aerated and "open" during the entire respiratory cycle (red dotted line). In the left lateral position $(L L)$, the entire right upper lung has the chance to open up at a low plateau pressures even under standard ventilator settings (gray lung). Once open, this right lung can maintain its "open lung" condition when turned to the right lateral positioning (RL) provided sufficient PEEP is applied. Notice the larger vertical distance in the lateral position required a higher PEEP to counteract the potential decrease in Ptp in the dependent lung. Now, the left lung is being recruited as it is placed in the uppermost gravity non-dependent position. At the end of the postural recruitment maneuver both lungs are free from atelectasis although the patient has returned to the baseline supine position 
monitoring lung aeration during and after lung recruitment maneuvers in real-time fashion [14-16].

\section{Effects of postural lung recruitment}

We analyzed three anesthetized children undergoing open inguinal hernia repair. Children aged 12, 24 and 34 months weighing 10,12 and $18 \mathrm{~kg}$, respectively. After approval by the local Ethical Committee and after obtaining Informed Consent from the parents, patients received general anesthesia under standard monitoring. Their lungs were ventilated in a pressure-controlled mode using an Aespire 7900 (Datex-Ohmeda, GE Healthcare, Helsinki, Finland) with the inspiratory pressure set to achieve a tidal volume of $7 \mathrm{~mL} / \mathrm{kg}$ (approx. 12 $\mathrm{cmH}_{2} \mathrm{O}$ ) and a respiratory rate adjusted between 20 and $25 \mathrm{bpm}$ to keep end-tidal of carbon dioxide between 35 and $40 \mathrm{mmHg}$. PEEP was $5 \mathrm{cmH}_{2} \mathrm{O}$, I:E 1:1 and $\mathrm{FIO}_{2} 0.5$.

Lung aeration was assessed by LUS using the linear 7-12 MHz transducer of the Esaote (MyLab Gamma, Genova, Italy). After anesthesia induction a standard bilateral LUS examination was performed in the supine position. Thereafter the echo probe was placed in an oblique direction between the ribs in the most dependent position as previously described [5]. All patients presented subpleural consolidations, air bronchograms and B-lines as signs of atelectasis (Figs. 2, 3).

Each patient was subjected to the following protocol sequence (Fig. 2):

- Testing the reaerating effect of $10 \mathrm{cmH}_{2} \mathrm{O}$ of PEEP After anesthesia induction in the supine position, PEEP was increased from 5 to $10 \mathrm{cmH}_{2} \mathrm{O}$ keeping ventilation constant for $3 \mathrm{~min}$. Then, ventilation returned to baseline settings but keeping PEEP at $8 \mathrm{cmH}_{2} \mathrm{O}$ to maintain any potential recruitment that $10 \mathrm{cmH}_{2} \mathrm{O}$ of PEEP may have induced.

- Testing the effect of postural recruitment Before emergence from anesthesia, patients were placed in the left lateral decubitus position for $90 \mathrm{~s}$ at constant ventilation but raising PEEP to $10 \mathrm{cmH}_{2} \mathrm{O}$. Thereafter, patients were turned on their right side for another $90 \mathrm{~s}$. At the end of the 3rd min, patients were placed back in the supine position returning to baseline ventilation, however, at a PEEP of $8 \mathrm{cmH}_{2} \mathrm{O}$ to keep the lungs open.

Figure 2 depicts the results of the protocol in one patient (case 1-24 months). LUS images taken at $8 \mathrm{cmH}_{2} \mathrm{O}$ of PEEP show that 3 min of ventilation with $10 \mathrm{cmH}_{2} \mathrm{O}$ of PEEP in the supine position did not have any measurable recruitment effect. Atelectasis persisted during and after PEEP elevation in all patients. Figure 3 shows the effect of the P-RM in the other two patients

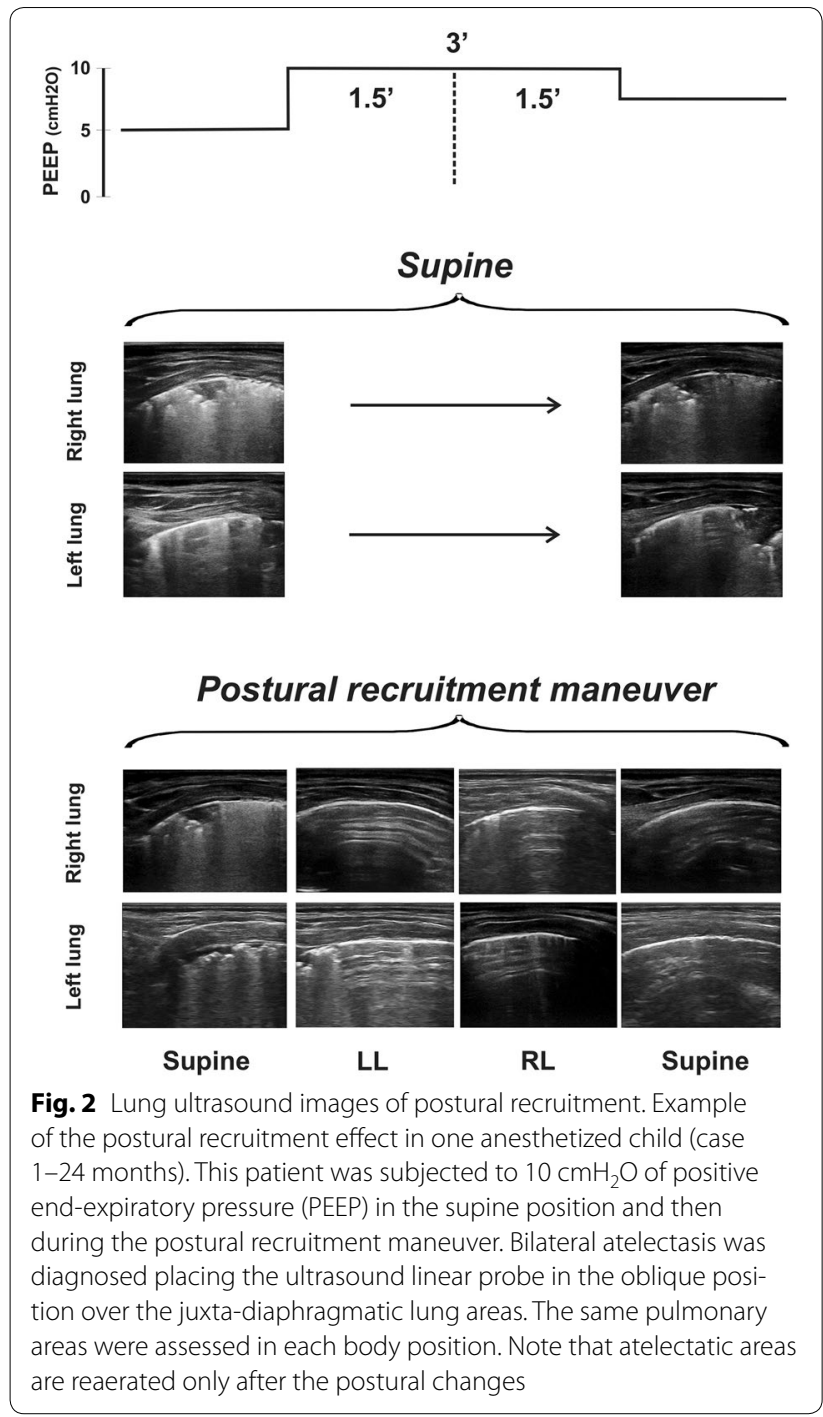

(case 2-12 months and case 3-34 months). The Additional file 1: video S1, Additional file 2: video S2 and Additional file 3: video S3 show the corresponding results than Figs. 2 and 3.

By contrast, the changing body positions of the P-RM clearly restored lung aeration in the atelectatic areas at the same ventilator settings. Reaeration of previously collapsed dependent lung areas was always observed as soon as they became non-dependent in the uppermost position; first in the right lung with the patient lying in the left lateral position and then in the left one with the patient in the right lateral decubitus. After the P-RM, both lungs remained free from atelectasis in the final supine position (Figs. 2, 3; Additional file 1: video S1, Additional file 2: video S2 and Additional file 3: video S3). Hemodynamics maintained stable throughout the protocol. 

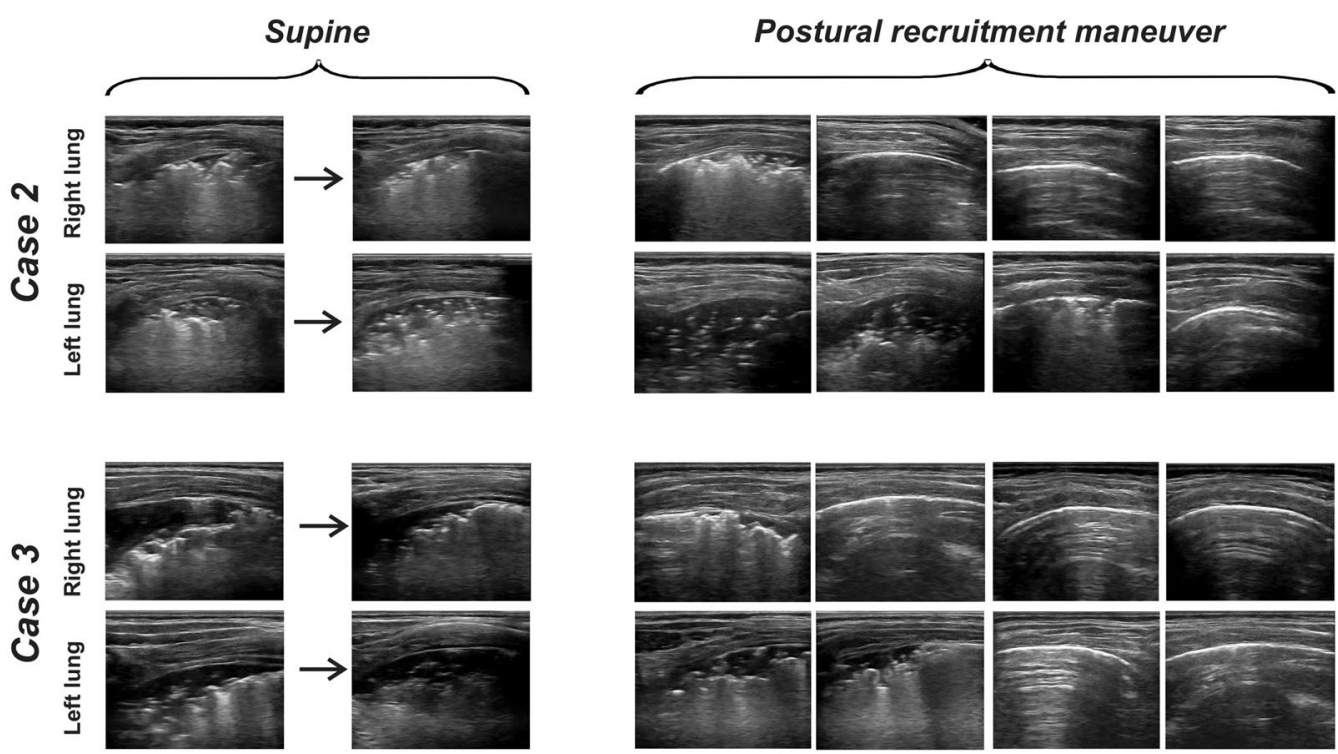

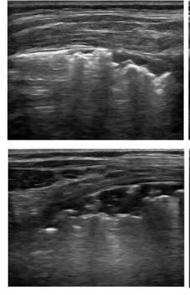

Supine

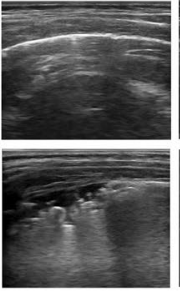

LL

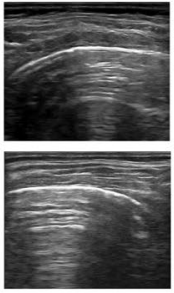

RL

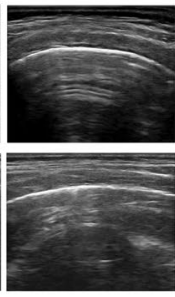

Supine

Fig. 3 Lung ultrasound images of postural recruitment in case 2 and 3

\section{Commentary}

We present LUS images documenting the effects of postural lung recruitment in children. Sequential changes in body position during constant ventilation at PEEP $10 \mathrm{cmH}_{2} \mathrm{O}$ decreased atelectasis even without applying the high airways pressures of classical recruitment maneuvers.

The therapeutic effect of P-RM seems to result from an increased Ptp caused by a gravity-dependent decrease in pleural pressure in the dorsal lungs and not by elevated airway pressures. As the right-to-left thoracic diameter is much larger than the anterior-posterior one, when a patient is placed in the lateral position, the resulting inspiratory Ptp within the uppermost lung will increase reaching the effective opening pressure of the collapsed lung tissue located in this region (Fig. 1). After the P-RM, a PEEP of $8 \mathrm{cmH}_{2} \mathrm{O}$ was enough to maintain an "open lung" condition in these three healthy patients [11].

The therapeutic implications of P-RM can be derived from the known negative consequence that atelectasis have on mechanically ventilated lungs. Perioperative respiratory complications such as hypoxemic events, bacterial translocation and pneumonia [17-21] adversely affect patient's outcomes while increasing health care cost in both, adults and pediatric patients $[22,23]$. Lung recruitment maneuvers easily overcome atelectasis in children with healthy and sick lungs alike $[3,13]$. They quickly abort hypoxemic events and could have some protective effect in atelectasis-mediated pneumonias [17, 24, 25].
However, the main objections against recruitment maneuvers are related to their potential hemodynamic side effects, especially in children. Reflexes triggered by high intrathoracic pressures cause bradycardia, compression of pulmonary capillaries and reduced venous return. Each of these either acting in isolation or combined are responsible for the hemodynamic impairment frequently encountered during recruitment maneuvers, predominantly in hypovolemic patients. In view of such undesirable consequences, the P-RM introduced in this paper presents an effective and efficient low risk alternative intervention for treating atelectasis in children. Turning the patients while applying $10 \mathrm{cmH}_{2} \mathrm{O}$ of PEEP for just 3 min seems to be uncritical in normovolemic children.

As far as we know, a postural lung recruitment maneuver has never before been described in children, although we found studies related to the effect of body positioning on respiratory function of mechanically ventilated patients. Some studies describe positive effects of lateral and prone positioning on respiratory function [26, 27] while others do not [28-30]. In these studies, body position was changed leaving ventilatory settings and PEEP constant. The lack of adequate PEEP adaptations-an essential therapeutic intervention of any recruitment strategy such as ours-may have led to these contradictory results. We reasoned that a slightly elevated PEEP should be applied for two reasons: (1) to obtain a plateau pressure high enough to reach the opening pressure of the uppermost lung areas and (2) to maintain the new 
recruited regions open when returning the patient to the supine position.

\section{Conclusions}

In this short communication, we present LUS images documenting the positive effects of postural recruitment in children. Changes in body position during constant ventilation at $10 \mathrm{cmH}_{2} \mathrm{O}$ of PEEP decreased atelectasis without the need to elevate airway pressures as in conventional recruitment maneuvers. Bedside imaging techniques such as LUS can help identify the best settings for a P-RM adapting them to the individual patient.

\section{Additional files}

Additional file 1: Video S1. Lung ultrasound images (LUS) of the isolated effect of $10 \mathrm{cmH}_{2} \mathrm{O}$ of PEEP and postural recruitment maneuvercase 1.

Additional file 2: Video S2. Lung ultrasound images (LUS) of the isolated effect of $10 \mathrm{cmH}_{2} \mathrm{O}$ of PEEP and postural recruitment maneuvercase 2.

Additional file 3: Video S3. Lung ultrasound images (LUS) of the isolated effect of $10 \mathrm{cmH}_{2} \mathrm{O}$ of PEEP and postural recruitment maneuvercase 3 .

\section{Authors' contributions}

GT and CMA made the clinical cases. GT, CMA, SHB, ADW, CF, MPM and FSS worked on the interpretation of the findings and in the final edition of the manuscript. All authors read and approved the final manuscript.

\section{Author details}

${ }^{1}$ Department of Anesthesiology, Hospital Privado de Comunidad, 7600 Mar del Plata, Buenos Aires, Argentina. ${ }^{2}$ Hepa Wash GmbH, Munich, Germany. ${ }^{3}$ Swisstom AG, Landquart, Switzerland. ${ }^{4}$ Department of Anesthesiology, University Hospital Valencia, Valencia, Spain. ${ }^{5}$ Department of Intensive Care Medicine, Instituto de Investigación Sanitaria Fundación Jiménez Díaz, IIS-FJD, Madrid, Spain. ${ }^{6}$ Hedenstierna Laboratory, Department of Surgical Sciences, Section of Anesthesia and Critical Care, Uppsala University Hospital, Uppsala, Sweden. ${ }^{7}$ CIBERES, Madrid, Spain. ${ }^{8}$ Department of Critical Care, Hospital La Fe, Valencia, Spain.

\section{Acknowledgements}

Not applicable.

\section{Competing interests}

The authors declare that they have no competing interests.

\section{Availability of data and materials}

Not applicable.

\section{Consent for publication}

A copy of the written consent is available for review by the editor-in-chief of this journal.

\section{Ethics approval and consent to participate}

The institutional review board (CIREI) approved this study, its publication and the corresponding written informed consent was obtained from the patient's relatives.

\section{Funding}

Author's own work.

\section{Publisher's Note}

Springer Nature remains neutral with regard to jurisdictional claims in published maps and institutional affiliations.

Received: 27 July 2017 Accepted: 20 September 2017

Published online: 13 October 2017

\section{References}

1. Damgaard-Pedersen K, Qvist T (1980) Pediatric pulmonary CT-scanning: anesthesia-induced changes. Pediatr Radiol 9:145-148

2. Sargent MA, McEachern AM, Jamieson DH, Kahwaji R (1999) Atelectasis on pediatric chest CT: comparison of sedation techniques. Pediatr Radiol 29:509-513

3. Tusman G, Bohm SH, Tempra A, Melkun F, García E, Turchetto E, Mulder PG, Lachmann B (2003) Effects of recruitment maneuver on atelectasis in anesthetized children. Anesthesiology 98:14-22

4. Lutterbey G, Wattjes MP, Doerr D, Fischer NJ, Gieseke J, Schild HH (2007) Atelectasis in children undergoing either propofol infusion or positive pressure ventilation anesthesia for magnetic resonance imaging. Pediatr Anesth 17:121-125

5. Acosta CM, Maidana GA, Jacovitti D, Belaunzaran A, Cereceda S, Rae E, Molina A, Gonorazky S, Böhm SH, Tusman G (2014) Accuracy of transthoracic lung ultrasound for diagnosing anesthesia-induced atelectasis in children. Anesthesiology 120:1370-1379

6. Froese AB, Bryan AC (1974) Effects of anesthesia and paralysis on diaphragmatic mechanics in man. Anesthesiology 41:242-255

7. Brismar B, Hedenstierna G, Lundquist H, Strandberg A, Svensson L, Tokics $L$ (2002) Pulmonary densities during anaesthesia with muscular relation: a proposal of atelectasis. Anesthesiology 62:422-428

8. Motoyama EK, Finder JD (2010) Respiratory physiology in infants and children. Smith's Anesth Infants Child Expert Consult Prem 5(6):22

9. Taussig LM, Hanis TR, Lebowitz MD (1977) Lung function in infants and young children: functional residual capacity, tidal volume, respiratory rate. Am Rev Respir Dis 116:233-239

10. Hedenstierna G, Edmark L (2010) Mechanisms of atelectasis in the perioperative period. Best Pract Res Clin Anaesthesiol 24:157-169

11. Lachmann B (1992) Open up the lung and keep the lung open. Intensive Care Med 18:319-321

12. Tusman G, Böhm SH (2010) Prevention and reversal of lung collapse during the intra-operative period. Best Pract Res Clin Anaesthesiol 24:183-197

13. Duff JP, Rosychuk RJ, Joffe AR (2007) The safety and efficacy of sustained inflations as a lung recruitment maneuver in pediatric intensive care unit patients. Intensive Care Med 33:1778-1786

14. Bouhemad B, Brisson H, Le-Guen M, Arbelot C, Lu Q, Rouby JJ (2015) Bedside ultrasound assessment of positive end-expiratory pressure-induced lung recruitment. Am J Respir Crit Care Med 183:341-347

15. Song IK, Kim EH, Lee JH (2016) Effects of an alveolar recruitment manoeuvre guided by lung ultrasound on anaesthesia-induced atelectasis in infants: a randomised, controlled trial. Anaesthesia. doi:10.1111/ anae. 13713

16. Tusman G, Acosta CM, Costantini M (2016) Ultrasonography for the assessment of lung recruitment maneuvers. Crit Ultrasound J 8:8

17. Scohy TV, Bikker IG, Hofland J, De JONG, Peter L, Bogers AJ, Gommers D (2009) Alveolar recruitment strategy and PEEP improve oxygenation, dynamic compliance of respiratory system and end-expiratory lung volume in pediatric patients undergoing cardiac surgery for congenital heart disease. Pediatr Anesth 19:1207-1212

18. Tusman G, Böhm SH, Warner DO, Sprung J (2012) Atelectasis and perioperative pulmonary complications in high-risk patients. Curr Opin Anesthesiol 25:1-10

19. Van Kaam AH, Lachmann RA, Herting E, De Jaegere A, Van Iwaarden F, Noorduyn LA, Kok JH, Haitsma JJ, Lachmann B (2004) Reducing atelectasis attenuates bacterial growth and translocation in experimental pneumonia. Am J Respir Crit Care Med 169:1046-1053

20. Brooks-Brunn JA (1995) Postoperative atelectasis and pneumonia. Heart Lung 24:94-115 
21. Elward AM, Warren DK, Fraser VJ (2002) Ventilator-associated pneumonia in pediatric intensive care unit patients: risk factors and outcomes. Pediatrics 109:758-764

22. Canet J, Mazo V (2010) Postoperative pulmonary complications. Minerva Anestesiol 76:138-143

23. Murat I, Constant I, Maud'Huy H (2004) Perioperative anaesthetic morbidity in children: a database of 24.165 anaesthetics over a 30-month period. Paediatr Anaesth 14:158-166

24. Lachmann RA, van Kaam AH, Haitsma JJ, Lachmann B (2007) High positive end-expiratory pressure levels promote bacterial translocation in experimental pneumonia. Intensive Care Med 33:1800-1804

25. Futier E, Constantin JM, Paugam-Burtz C, Pascal J, Eurin M, Neuschwander A, Allaouchiche B (2013) A trial of intraoperative low-tidal-volume ventilation in abdominal surgery. N Engl J Med 369:428-437
26. Remolina C, Khan A, Santiago T, Edelman NH (1981) Positional hypoxemia in unilateral lung disease. N Engl J Med 304:523-525

27. Chang SC, Chang HI, Shiao GM, Perng RP (1993) Effect of body position gas exchange in patients with unilateral central airway lesions: down with the good lung? Chest 103:787-791

28. Montgomery K, Choy NL, Steele M, Hough J (2014) The effectiveness of quarter turn from prone in maintaining respiratory function in premature infants. J Paediatr Child Health 50:972-977

29. Balaguer A, Escribano J, Roque M (2006) Infant position in neonates receiving mechanical ventilation. Cochrane Database Syst Rev 4:68

30. Numa AH, Hammer J, Newth CJL (1997) Effect of prone and supine positions on functional residual capacity, oxygenation, and respiratory mechanics in ventilated infants and children. Am J Respir Crit Care Med $156: 1185-1189$

\section{Submit your manuscript to a SpringerOpen ${ }^{\odot}$ journal and benefit from:}

- Convenient online submission

- Rigorous peer review

- Open access: articles freely available online

- High visibility within the field

- Retaining the copyright to your article

Submit your next manuscript at $\boldsymbol{\nabla}$ springeropen.com 